$11-1-2013$

\title{
Test for Intraclass Correlation Coefficient under Unequal Family Sizes
}

\author{
Madhusudan Bhandary \\ Columbus State University, Columbus, GA, bhandary_madhusudan@colstate.edu
}

Koji Fujiwara

North Dakota State University, Fargo, ND, koji.fujiwara@ndsu.edu

Follow this and additional works at: http://digitalcommons.wayne.edu/jmasm

Part of the Applied Statistics Commons, Social and Behavioral Sciences Commons, and the Statistical Theory Commons

\section{Recommended Citation}

Bhandary, Madhusudan and Fujiwara, Koji (2013) "Test for Intraclass Correlation Coefficient under Unequal Family Sizes," Journal of Modern Applied Statistical Methods: Vol. 12 : Iss. 2 , Article 9.

DOI: $10.22237 /$ jmasm/1383278880

Available at: http://digitalcommons.wayne.edu/jmasm/vol12/iss2/9

This Regular Article is brought to you for free and open access by the Open Access Journals at DigitalCommons@WayneState. It has been accepted for inclusion in Journal of Modern Applied Statistical Methods by an authorized editor of DigitalCommons@WayneState. 


\section{Test for Intraclass Correlation Coefficient under Unequal Family Sizes}

\author{
Madhusudan Bhandary \\ Columbus State University \\ Columbus, GA
}

\author{
Koji Fujiwara \\ North Dakota State University \\ Fargo, ND
}

Three tests are proposed based on F-distribution, Likelihood Ratio Test (LRT) and large sample Z-test for intraclass correlation coefficient under unequal family sizes based on a single multinormal sample. It has been found that the test based on F-distribution consistently and reliably produces results superior to those of Likelihood Ratio Test (LRT) and large sample Z-test in terms of size for various combinations of intraclass correlation coefficient values. The power of this test based on F-distribution is competitive with the power of the LRT and the power of Z-test is slightly better than the powers of F-test and LRT when $k=15$, but the power of Z-test is worse in comparison with the F-test and LRT for $k=30$, i.e. for large sample situation, where $k=$ sample size. This test based on F-distribution can be used for both small sample and large sample situations. An example with real data is presented.

Keywords: $\quad$ Likelihood ratio test, Z-test, F-test, intraclass correlation coefficient.

\section{Introduction}

Suppose it is required to estimate the correlation coefficient between blood pressures of children on the basis of measurements taken on $p$ children in each of $n$ families. The $p$ measurements on a family provide $p(p-1)$ pairs of observations, $(x, y)$--- $x$ being the blood pressure of one child and $y$ that of another. From the $n$ families a total of $n p(p-1)$ pairs are generated from which a correlation coefficient is computed in the ordinary way.

The correlation coefficient thus computed is called an intraclass correlation coefficient. It is important to have statistical inference concerning intraclass correlation, because it provides information regarding blood pressure, cholesterol, etc., in a family within some race in the world.

Madhusudan Bhandary is a Professor in the Department of Mathematics. Email him at: bhandary_madhusudan@colstate.edu.Koji Fujiwara is a graduate student in the Department of Statistics. Email him at: koji.fujiwara@ndsu.edu. 


\section{TEST FOR INTRACLASS CORRELATION COEFFICIENT}

The intraclass correlation coefficient $\rho$ has a wide variety of uses in measuring the degree of intrafamily resemblance with respect to characteristics such as blood pressure, cholesterol, weight, height, stature, lung capacity, etc.

Several authors have studied statistical inference concerning $\rho$ based on a single multinormal sample ( Scheffe, 1959; Rao, 1973; Rosner et.al, 1977, 1979,; Donner and Bull, 1983; Srivastava, 1984; Konishi, 1985; Gokhale and SenGupta, 1986; SenGupta, 1988; Velu and Rao, 1990). Donner and Bull (1983) discussed the likelihood ratio test for testing the equality of two intraclass correlation coefficients based on two independent multinormal samples under equal family sizes. Konishi and Gupta (1987) proposed a modified likelihood ratio test and derived its asymptotic null distribution. They also discussed another test procedure based on a modification of Fisher's Z-transformation following Konishi (1985).

Huang and Sinha (1993) considered an optimum invariant test for the equality of intraclass correlation coefficients under equal family sizes for more than two intraclass correlation coefficients based on independent samples from several multinormal distributions. For unequal family sizes, Young and Bhandary (1998) proposed Likelihood ratio test, large sample Z-test and large sample Z* test for the equality of two intraclass correlation coefficients based on two independent multinormal samples.

For several populations and unequal family sizes, Bhandary and Alam (2000) proposed Likelihood ratio test and large sample ANOVA test for the equality of several intraclass correlation coefficients based on several independent multinormal samples. Donner and Zou (2002) proposed asymptotic test for the equality of dependent intraclass correlation coefficients under unequal family sizes.

However, none of the above authors derived any test for a single sample and unequal family sizes. It is an important practical problem to consider a single sample test for intraclass correlation coefficient under unequal family sizes.

This article considers three tests for intraclass correlation coefficient based on a single multinormal sample under unequal family sizes. Conditional analysis is conducted assuming family sizes fixed though unequal. It could be of interest to examine the blood pressure or cholesterol or lung capacity, etc., among families in U.S.A. or among some other races, and therefore it is necessary to develop a single sample test for intraclass correlation coefficient under unequal family sizes.

Three tests are proposed: F-test, LRT and large sample Z-test. These three tests are compared in Section 3 using simulation technique. It has been found on the basis of simulation study that the test based on F-distribution consistently and 


\section{BHANDARY \& FUJIWARA}

reliably produced results superior to those of Likelihood Ratio Test (LRT) and large sample Z-test in terms of size for various combinations of intraclass correlation coefficient values.

The power of this test based on F-distribution is competitive with the power of the LRT, and the power of Z-test is slightly better than the powers of F-test and LRT when $k=15$; but the power of Z-test is worse in comparison with the F-test and LRT for $k=30$, i.e. for large sample situation, where $k=$ sample size.

This test based on F-distribution can be used for both small sample and large sample situations.

An example with real data is presented in Section 4.

\section{Tests of $H_{0} \rho=\rho \quad$ Versus $H_{1} \rho^{1} \rho \quad 0$}

\section{Likelihood Ratio Test:}

Let $X_{i}=\left(x_{i 1}, x_{i 2}, \ldots, x_{i p_{i}}\right)^{\prime}$ be a $p_{i} x 1$ vector of observations from $i^{\text {th }}$ family;

$$
i=1,2, \ldots, k \text {. }
$$

The structure of mean vector and the covariance matrix for the familial data is given by the following ( Rao 1973) :

$$
\underset{\sim}{\mu} \mu_{i}=\mu 1_{\sim} \text { and } \underset{p_{i} x p_{i}}{\Sigma_{i}}=\sigma^{2}\left(\begin{array}{cccc}
1 & \rho & \ldots & \rho \\
\rho & 1 & \ldots & \rho \\
\ldots & \ldots & \ldots & \ldots \\
\rho & \rho & \ldots & 1
\end{array}\right)
$$

where $1_{i}$ is a $p_{i} x 1$ vector of 1 's, $\mu(-\infty<\mu<\infty)$ is the common mean and $\sigma^{2}\left(\sigma^{2}>0\right)$ is the common variance of members of the family and $\rho$, which is called the intraclass correlation coefficient, is the coefficient of correlation among the members of the family and $\max _{1 \leq i \leq k}\left(-\frac{1}{p_{i}-1}\right) \leq \rho \leq 1$.

It is assumed that $x_{i} \sim N_{p_{i}}\left(\mu_{i}, \Sigma_{i}\right) ; i=1, \ldots, k$, where $N_{p_{i}}$ represents $p_{i}$ variate normal distribution and $\mu_{i}, \Sigma_{i}$ 's are defined in (1). 


\section{TEST FOR INTRACLASS CORRELATION COEFFICIENT}

Let $u_{i}=\left(u_{i 1}, u_{i 2}, \ldots, u_{i p_{i}}\right)^{\prime}=Q x_{i}$

where $Q$ is an orthogonal matrix.

Under the orthogonal transformation (2), it can be seen that $u_{i} \sim N_{p_{i}}\left(\mu_{i}^{*}, \Sigma_{i}^{*}\right) ; i=1, \ldots, k$

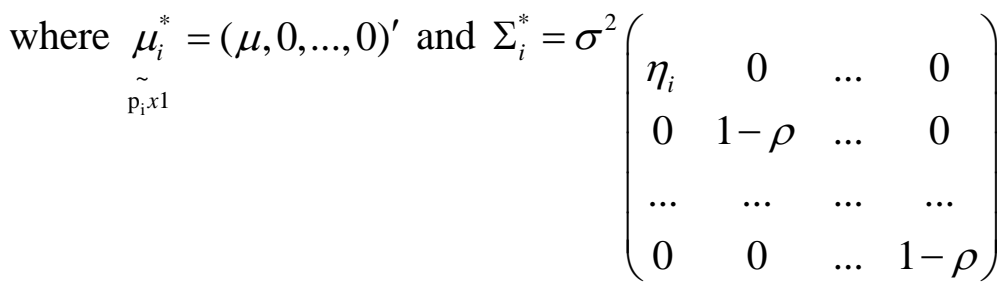

and $\eta_{i}=p_{i}^{-1}\left\{1+\left(p_{i}-1\right) \rho\right\}$

The transformation used on the data from $x$ to $u$ above is independent of $\rho$. One can use Helmert's orthogonal transformation.

Srivastava (1984) gives the estimator of $\rho$ and $\sigma^{2}$ under unequal family sizes which are good substitutes for the maximum likelihood estimators and are given by the following:

$$
\begin{gathered}
\hat{\rho}=1-\frac{\hat{\gamma}^{2}}{\hat{\sigma}^{2}} \\
\hat{\sigma}^{2}=(k-1)^{-1} \sum_{i=1}^{k}\left(u_{i 1}-\hat{\mu}\right)^{2}+k^{-1} \hat{\gamma}^{2}\left(\sum_{i=1}^{k} a_{i}\right) \\
\hat{\gamma}^{2}=\frac{\sum_{i=1}^{k} \sum_{r=2}^{p_{i}} u_{i r}^{2}}{\sum_{i=1}^{k}\left(p_{i}-1\right)} \\
\hat{\mu}=k^{-1} \sum_{i=1}^{k} u_{i 1}
\end{gathered}
$$

and $a_{i}=1-p_{i}^{-1}$

Now, consider a random sample of $k$ families from a population. 


\section{BHANDARY \& FUJIWARA}

Let $x_{i}=\left(x_{i 1}, x_{i 2}, \ldots, x_{i p_{i}}\right)^{\prime}$ be a $p_{i} x 1$ vector of observations from $i^{\text {th }}$ family; $i=1,2, \ldots, K$

and $\left.\underset{\sim}{x_{i}} \sim N_{p_{i}} \underset{\sim}{\left(\mu_{i}\right.}, \Sigma_{i}\right)$, where $\underset{\sim}{\mu_{i}}=\mu \underset{\sim}{1_{i}}, \Sigma_{i}=\sigma^{2}\left(\begin{array}{cccc}1 & \rho & \ldots & \rho \\ \rho & 1 & \ldots & \rho \\ \ldots & \ldots & \ldots & \ldots \\ \rho & \rho & \ldots & 1\end{array}\right)$

and $\max _{1 \leq i \leq k_{1}}\left(-\frac{1}{p_{i}-1}\right) \leq \rho \leq 1$.

Using orthogonal transformation, the data vector can be transformed from $x_{i}$ to $u_{i}$ as follows:

$\underset{\sim}{u_{i}}=\left(u_{i 1}, u_{i 2}, \ldots, u_{i p_{i}}\right)^{\prime} \sim N_{p_{i}}\left(\underset{\sim}{\mu_{i}^{*}}, \Sigma_{i}^{*}\right) ; i=1, \ldots, k$

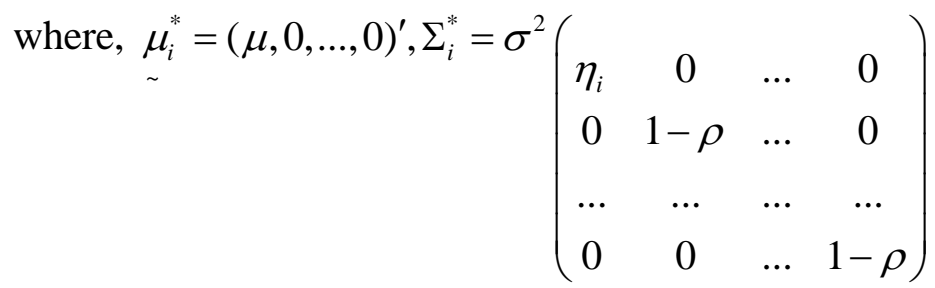

$\eta_{i}=p_{i}^{-1}\left\{1+\left(p_{i}-1\right) \rho\right\}$

The transformation used on the data above from $x$ to $u$ is independent of $\rho$.

Under the above setup, likelihood ratio test statistic for testing $H_{0}: \rho=\rho_{0}$ Vs. $H_{1}: \rho \neq \rho_{0}$ is given by the following:

$-2 \log \Lambda=\sum_{i=1}^{k} \log \left[p_{i}^{-1}\left\{1+\left(p_{i}-1\right) \rho_{0}\right\}\right]+\sum_{i=1}^{k}\left(p_{i}-1\right) \log \left(1-\rho_{0}\right)$ 


$$
\begin{aligned}
& +\frac{1}{\hat{\sigma}^{2}}\left[\sum_{i=1}^{k}\left\{p_{i}\left(u_{i 1}-\hat{\mu}\right)^{2} /\left[1+\left(p_{i}-1\right) \rho_{0}\right]\right\}+\sum_{i=1}^{k} \sum_{r=2}^{p_{i}} u_{i r}^{2} /\left(1-\rho_{0}\right)\right] \\
& -\sum_{i=1}^{k} \log \left[p_{i}^{-1}\left\{1+\left(p_{i}-1\right) \hat{\rho}\right\}\right]-\sum_{i=1}^{k}\left(p_{i}-1\right) \log (1-\hat{\rho}) \\
& -\frac{1}{\hat{\sigma}^{2}}\left[\sum_{i=1}^{k}\left\{p_{i}\left(u_{i 1}-\hat{\mu}\right)^{2} /\left[1+\left(p_{i}-1\right) \hat{\rho}\right]\right\}+\sum_{i=1}^{k} \sum_{r=2}^{p_{i}} u_{i r}^{2} /(1-\hat{\rho})\right]
\end{aligned}
$$

where, $\Lambda$ = likelihood ratio test statistic,

$$
\begin{aligned}
& \hat{\rho}=\text { estimate of intraclass correlation coefficient under } H_{1} \text {, } \\
& \hat{\sigma}^{2}=\text { estimate of } \sigma^{2}
\end{aligned}
$$

and $\hat{\mu}$ is the estimate of mean.

The estimators $\hat{\rho}, \hat{\sigma}^{2}$ and $\hat{\mu}$ can be obtained from Srivastava's estimator given by (3).

It is well-known from asymptotic theory that $-2 \log \Lambda$ has an asymptotic chi-square distribution with 1 degree of freedom.

\section{Large Sample Z-test:}

A large sample Z-test is proposed as follows :

$$
Z=\frac{\hat{\rho}-\rho_{0}}{\sqrt{\frac{V a r}{k}}}
$$

where, $\hat{\rho}=$ the estimator of $\rho$ from the sample using Srivastava (1984) and Var under $H_{0}$ ( using Srivastava and Katapa (1986) ) is as follows :

$\operatorname{Var}=2\left(1-\rho_{0}\right)^{2}\left\{(\bar{p}-1)^{-1}+c^{2}-2\left(1-\rho_{0}\right)(\bar{p}-1)^{-1} k^{-1}\left(\sum_{i=1}^{k} a_{i}\right)\right\}$

where, $k=$ number of families in the sample 


$$
\begin{aligned}
& \bar{p}=k^{-1} \sum_{i=1}^{k} p_{i} \\
& c^{2}=1-2\left(1-\rho_{0}\right)^{2} k^{-1} \sum_{i=1}^{k} a_{i}+\left(1-\rho_{0}\right)^{2}\left[k^{-1} \sum_{i=1}^{k} a_{i}+(\bar{p}-1)^{-1} \bar{a}^{2}\right] \\
& \bar{a}=k^{-1} \sum_{i=1}^{k} a_{i}
\end{aligned}
$$

and $a_{i}=1-p_{i}^{-1}$

It is obvious ( using Srivastava and Katapa (1986) ) to see that under $H_{0}$, the test statistic $Z$ given by ( 7$)$ has an asymptotic $N(0,1)$ distribution. Because the alternative hypothesis is $H_{1}: \rho \neq \rho_{0}$, the above Z-test is a two sided test.

\section{F-test:}

Using (5), $u_{i 1} \sim N_{1}\left(\mu, \sigma^{2} \eta_{i}\right)$

where $\eta_{i}=p_{i}^{-1}\left\{1+\left(p_{i}-1\right) \rho\right\}$

Hence $\sum_{i=1}^{k} \frac{\left(u_{i 1}-\hat{\mu}\right)^{2}}{\sigma^{2} \eta_{i}} \sim \chi_{k-1}^{2}$

Also from (5),

$$
\frac{\sum_{i=1}^{k} \sum_{r=2}^{p_{i}} u_{i r}^{2}}{\sigma^{2}(1-\rho)} \sim \chi_{\sum_{i=1}^{k}\left(p_{i}-1\right)}^{2}
$$

and $\sum_{i=1}^{k} \frac{\left(u_{i 1}-\hat{\mu}\right)^{2}}{\sigma^{2} \eta_{i}}$ and $\frac{\sum_{i=1}^{k} \sum_{r=2}^{p_{i}} u_{i r}^{2}}{\sigma^{2}(1-\rho)}$ are independent.

$\chi_{n}^{2}$ denotes Chi-square distribution with $\mathrm{n}$ degrees of freedom.

Hence, an F-test is proposed as: 


$$
\begin{aligned}
& F=\frac{\sum_{i=1}^{k} \frac{\left(u_{i 1}-\hat{\mu}\right)^{2}}{p_{i}^{-1}\left\{1+\left(p_{i}-1\right) \rho_{0}\right\}} /(k-1)}{\frac{\sum_{i=1}^{k} \sum_{r=2}^{p_{i}} u_{i r}^{2}}{\left(1-\rho_{0}\right)} /\left(\sum_{i=1}^{k}\left(p_{i}-1\right)\right)} \\
& \text { Under } H_{0}, F \sim F_{k-1, \sum_{i=1}^{k}\left(p_{i}-1\right)}
\end{aligned}
$$

The performance of the three tests given by (6), (7) and (9) is discussed in terms of size and power in the next section using simulated data.

\section{Simulation Results:}

Multivariate normal random vectors were generated using $\mathrm{R}$ program in order to evaluate the power of the F statistic as compared to the LRT statistic and Zstatistic. Five and thirty vectors of family data were created for the population. The family size distribution was truncated to maintain the family size at a minimum of 2 siblings and a maximum of 15 siblings. The previous research in simulating family sizes ( Rosner et al. (1977), Srivastava and Keen (1988) ) determined the parameter setting for FORTRAN IMSL negative binomial subroutine with a mean $=2.86$ and a success probability $=0.483$.

Here, a mean $=2.86$ and a theta $=41.2552$ were set.

All parameters were set the same except the value of $\rho$ which took on all combinations possible over the range of values from 0.1 to 0.9 at increments of 0.1 .

The R program produced estimates of $\rho$ along with F statistic, the LRT statistic and the Z- statistic 3,000 times for each particular population parameter $\rho$.

The frequency of rejection of each test statistic at $\alpha=0.05$ was noted and the proportion of rejections are calculated for various combination of $\rho$.

The sizes for the LRT statistic, F statistic and Z statistic for various combination of $\rho$ were also calculated.

On the basis of this study, it was found that the test based on F-distribution consistently and reliably produced results superior to those of Likelihood Ratio Test (LRT) and large sample Z-test in terms of size for various combinations of intraclass correlation coefficient values. 


\section{BHANDARY \& FUJIWARA}

The power of this test based on F-distribution is competitive with the power of the LRT and the power of Z-test is slightly better than the powers of F-test and LRT when $k=15$ but the power of Z-test is worse in comparison with the F-test and LRT for $\mathrm{k}=30$ i.e. for large sample situation.

Hence, overall recommendation should be to use F-test given by (9).

The LRT has a large bias under $H_{0}$ and this bias is probably due to the fact that $-2 \log \Lambda$ may take some negative values (which it should not take) and those negative values are deleted for the calculation of size and power. But, for the Ftest or Z-test, this problem does not arise.

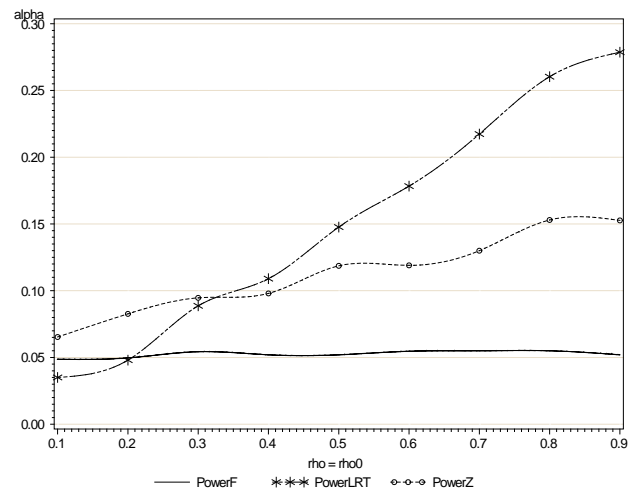

Figure 1. Alpha Levels $(k=15$, alpha $=$ 0.05)

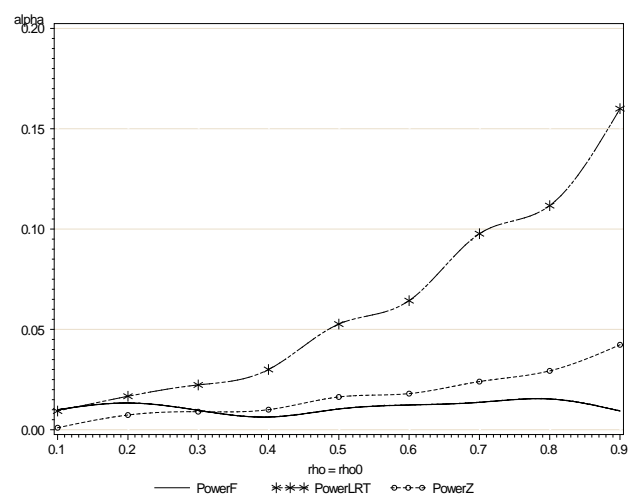

Figure 2. Alpha Levels $(k=30$, alpha $=$ $0.01)$

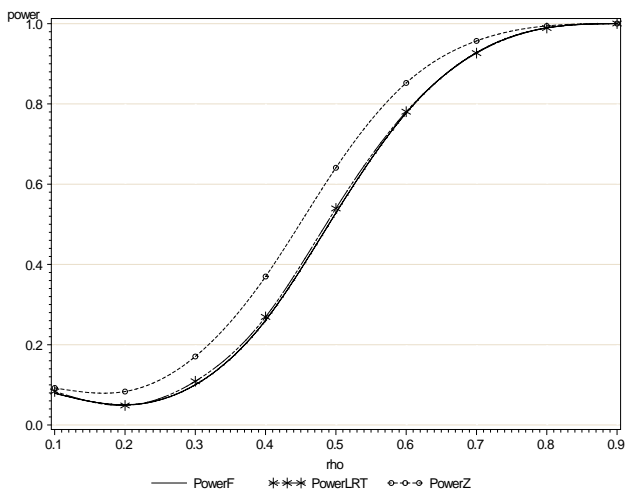

Figure 3. Power (alpha $=0.05, k=15$, rho0 $=0.2)$

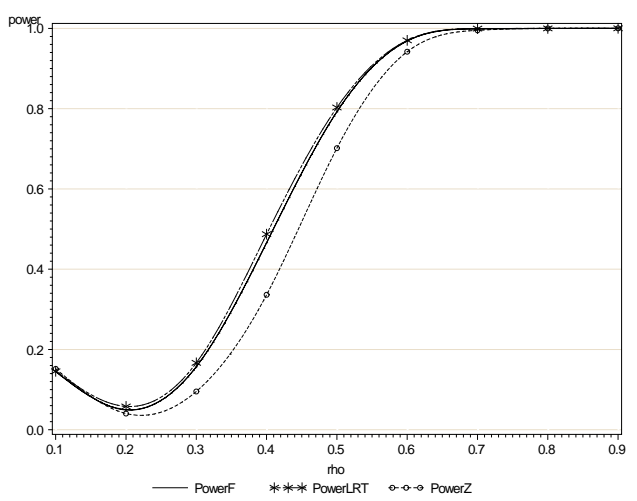

Figure 4. Power (alpha $=0.05, k=30$, rho0 $=0.2$ ) 


\section{TEST FOR INTRACLASS CORRELATION COEFFICIENT}

The test based on F-distribution can be used for both small sample and large sample situations.

Hence, the F test is strongly recommended for use in practice.

\section{Example with Real Life Data}

The three tests are compared using real life data collected from Srivastava and Katapa (1986). Table 1 gives the values of pattern intensity on soles of feet in fourteen families, where values for daughters and sons are put together. In the Table below, for example, for family \#11, the children (both sons and daughters) have feet sizes 5,3,4,4 respectively.

Table 1. Pattern intensity values on soles of feet for 14 families.

\begin{tabular}{lll}
\hline Sample & Family & Siblings \\
\hline A & 12 & 2,4 \\
A & 10 & $4,5,4$ \\
A & 9 & 5,6 \\
A & 1 & 2,2 \\
A & 4 & $2,2,2,2,2$ \\
A & 5 & 6,6 \\
A & 8 & $2,4,7,4,4,7,8$ \\
A & 3 & $2,2,2$ \\
A & 6 & $4,3,3$ \\
A & 14 & $2,2,2$ \\
A & 7 & $2,2,3,6,3,5,4$ \\
A & 2 & 2,3 \\
A & 11 & $5,3,4,4$ \\
A & 13 & $4,3,3,3$ \\
\hline
\end{tabular}

The data on the children from Table 1 was used to analyze the case of testing intraclass correlation coefficient. The above data were collected for the purpose of having inference on familial correlation. Srivastava and Katapa (1986) used the above data to estimate the intraclass correlation coefficient for unequal family sizes. 


\section{BHANDARY \& FUJIWARA}

First, the data is transformed by multiplying each observation vector by Helmert's orthogonal matrix $Q$

$$
\text { where, } \underset{p_{i} \times p_{i}}{Q}=\left[\begin{array}{ccccc}
\frac{1}{\sqrt{p_{i}}} & \frac{1}{\sqrt{p_{i}}} & \frac{1}{\sqrt{p_{i}}} & \ldots & \frac{1}{\sqrt{p_{i}}} \\
\frac{1}{\sqrt{2}} & -\frac{1}{\sqrt{2}} & 0 & \ldots & 0 \\
\frac{1}{\sqrt{6}} & \frac{1}{\sqrt{6}} & -\frac{2}{\sqrt{6}} & 0 & 0 \\
\ldots & \ldots & \ldots & \ldots & \ldots \\
\frac{1}{\sqrt{p_{i}\left(p_{i}-1\right)}} & \frac{1}{\sqrt{p_{i}\left(p_{i}-1\right)}} \frac{1}{\sqrt{p_{i}\left(p_{i}-1\right)}} & \ldots & -\frac{\left(p_{i}-1\right)}{\sqrt{p_{i}\left(p_{i}-1\right)}}
\end{array}\right]
$$

This results in transformed vectors $u_{i}$ for $i=1,2, \ldots, k$. Here, $k=14$.

Srivastava's formula given by (3) is used to compute intraclass correlation coefficient and variance. The computed values of intraclass correlation coefficient and variance are $\hat{\rho}=0.8118$ and $\hat{\sigma}^{2}=8.8578$. Because $\hat{\rho}=0.8118$ is estimated from the above sample, it is necessary to know whether the intraclass correlation coefficient $\rho$ in the population from which the sample came is close to 0.8 , and therefore necessary to test $H_{0}: \rho=0.8$ Vs. $H_{1}: \rho \neq 0.8$.

Formulae (6) and (7) and (9) are used to obtain the values of the test statistics for testing $H_{0}: \rho=0.8$ Vs. $H_{1}: \rho \neq 0.8$. The computed values of the LRT statistic, Z statistic and F statistic obtained from formula (6), (7) and (9) respectively are as follows:

LRT statistic $=331.31, \mathrm{Z}$ statistic $=0.10642$ and $\mathrm{F}$ statistic $=0.7316$

The critical values at $\alpha=0.05$ and 0.10 for the tests are as follows:

$$
\begin{aligned}
& L R T_{0.05}=3.8415 ; Z_{0.025}=1.96 ; F_{0.025}=2.8506 ; F_{0.975}=0.33037 ; \\
& L R T_{0.10}=2.7055 ; Z_{0.05}=1.645 ; F_{0.05}=2.3973 ; F_{0.95}=0.39763 .
\end{aligned}
$$

Hence, the null hypothesis is accepted by F- test and Z- test at 5\% and 10\% levels, whereas it is rejected by LRT at 5\% and 10\% levels. 


\section{TEST FOR INTRACLASS CORRELATION COEFFICIENT}

If the F-test is used it provides information that the population intraclass correlation coefficient is 0.8 which should be true intuitively. But, LRT gives information that the population intraclass correlation coefficient is not 0.8 . Therefore, it is important to choose the right test to produce the correct information. It was found before that LRT is biased in terms of size; therefore the recommendation is to use F-test given by (9) in real practice.

\section{References}

Bhandary, M., \& Alam, M. K. (2000). Test for the equality of intraclass correlation coefficients under unequal family sizes for several populations. Communications in Statistics-Theory and Methods, 29(4), 755-768.

Donner, A., \& Bull, S. (1983). Inferences concerning a common intraclass correlation coefficient. Biometrics, 39, 771-775.

Donner, A. , \& Zou, G. (2002). Testing the equality of dependent intraclass correlation coefficients. The Statistician, 51(3), 367-379.

Gokhale, D. V., \& SenGupta, A. (1986). Optimal tests for the correlation coefficient in a symmetric multivariate normal population. J. Statist. Plann Inference, 14, 263-268.

Huang, W., \& Sinha, B. K. (1993). On optimum invariant tests of equality of intraclass correlation coefficients. Annals of the Institute of Statistical Mathematics, 45(3), 579-597.

Konishi, S. (1985). Normalizing and variance stabilizing transformations for intraclass correlations. Annals of the Institute of Statistical Mathematics, 37, 8794.

Konishi, S. , \& Gupta, A. K. (1987). Testing the equality of several intraclass correlation coefficients. J. Statist. Plann. Inference, 21, 93-105.

Rao, C.R. (1973). Linear Statistical Inference and Its Applications. Wiley, New York.

Rosner, B., Donner, A., \& Hennekens, C.H. (1977). Estimation of intraclass correlation from familial data. Applied Statistics, 26, 179-187.

Rosner, B., Donner, A., \& Hennekens, C.H. (1979). Significance testing of interclass correlations from familial data. Biometrics, 35, 461-471.

Scheffe, H. (1959). The Analysis of Variance. Wiley, New York.

SenGupta, A. (1988). On loss of power under additional information - an example. Scand. J. Statist., 15, 25-31. 


\section{BHANDARY \& FUJIWARA}

Srivastava, M. S. (1984). Estimation of interclass correlations in familial data. Biometrika, 71, 177-185.

Srivastava, M.S. , \& Katapa, R. S. (1986). Comparison of estimators of interclass and intraclass correlations from familial data. Canadian Journal of Statistics, 14, 29-42.

Srivastava, M. S. , \& Keen, K. J. (1988). Estimation of the interclass correlation coefficient. Biometrika, 75, 731-739.

Velu, R. , \& Rao, M. B. (1990). Estimation of parent-offspring correlation. Biometrika, 77(3), 557-562.

Young, D., \& Bhandary, M. (1998). Test for the equality of intraclass correlation coefficients under unequal family sizes. Biometrics, 54(4), 1363-1373. 